\title{
The efficacy of IORT (intraoperative radiotherapy) for early advanced breast cancer depending on the time delay of external beam irradiation (EXRT) post conservative breast surgery (CBS)
}

\author{
Agata Celejewska', Bogusław Maciejewski², Jerzy Wydmański ${ }^{1}$ Krzysztof Składowski ${ }^{3}$ \\ 'Dept. Radiotherapy, M. Sklodowska-Curie National Research Institute of Oncology, Gliwice Branch, Gliwice, Poland \\ ${ }^{2}$ Div. Research Programmes, M. Sklodowska-Curie National Research Institute of Oncology, Gliwice Branch, Gliwice, Poland \\ ${ }^{3}$ Dept. Radiotherapy and Chemotherapy I, M. Sklodowska-Curie National Research Institute of Oncology, Gliwice Branch, Gliwice, Poland
}

Introduction. The following study focuses on the efficacy of the IORT ( $\left.14.4 \mathrm{izOGy}_{2.0}\right)$ as part of conservative surgery with adjuvant EXRT (50 Gy in 25 fractions) for low risk 109 early breast cancer patients and 106 intermediate or nonlinear high risk patients with adjuvant chemoradiation or chemotherapy followed by the EXRT.

Material and methods. The accumulation of the rates of local recurrences (LR) and distant metastases (DM) are characterized by nonlinear but two-phase curves.

Results. During the first 5 years, $67 \%$ of all LR, and only $43 \%$ of all DM occurred, and between the $8^{\text {th }}$ and $10^{\text {th }}$ years the LR curve steeply increases by $25 \%$ and the DM by $48 \%$.

Conclusion. This suggest that a 5 -year follow-up is too short and should be extended to 10 years. Among the analyzed prognostic factors, the time interval (TI) between IORT and adjuvant EXRT has occurred the major prognostic risk factor. If the TI is extended over 60 days (delayed EXRT), the LR and the DM risk undergoes a3-10 fold increase. Concurrent CH-EXRT significantly lowers local and distant failures, compared with delayed EXRT after completing CHT. Therefore, delayed EXRT completely ruins the expected efficacy of the IORT.

Key words: IORT, early breast cancer, delayed adjuvant EXRT

\section{Introduction}

Since the 1960s when Abe and Takahashi [1] presented the basic rationale and methods of intraoperative radiotherapy (IORT), this method has been widely used alone, or combined with external irradiation (EXRT) and/or chemotherapy (CHT) to improve the treatment outcomes of various malignant tumors, including breast cancer. The results of the IORT as a single therapy has been critically evaluated [2,3]. Reitsamer et al. [4] noted no local recurrences of breast cancer after IORT BOOST combined with EXRT, but the follow-up was too short. Herskind et al. [5-7], presenting the radiobiological aspects of the IORT, has focused on the biological advantages of this method. Recently, Fastner et al. [18] published a comprehensive overview of the role of the IORT as the ESTRO IORTTASK

\section{How to cite:}

Celejewska A, Maciejewski B, Wydmański J, Składowski K. The efficacy of IORT (intraoperative radiotherapy) for early advanced breast cancer depending on the time delay of external beam irradiation (EXRT) post conservative breast surgery (CBS). NOWOTWORY J Oncol 2021; 71: 133-138. 
Force/ACROP recommendations, suggesting this method as a favorable standard of combined therapy for carefully selected locally advanced breast cancer patients. Despite the many studies, it is not easy to interpret the results of various IORT doses combined with EXRT and/or chemotherapy. The majority of studies concentrate on local recurrence as the major end-point, but incidences of distant metastases are usually ignored. Moreover, adjuvant therapy post conservative surgery (CBS) includes various methods, i.e. concurrent chemoradiation, chemotherapy followed by EXRT or the reverse.

For high risk breast cancer patients, the IORT with CBS might not be effective enough mainly, when adjuvant EXRT is delayed, because among others processes, repopulation of the survived cancer cells accelerates, and therefore decreases or even thwarts the expected efficacy of the IORT. It seems that the longer the delay of postop. EXRT, the higher the risk of local recurrence. Because the prognostic importance of the time interval between CBS (IORT) and EXRT has not been evaluated yet, the present study is mainly focused on this topic.

\section{Material}

The retrospective clinical material consists of 215 consecutive breast cancer patients ( $(1-2 \mathrm{NO}-\mathrm{N}+$ ) treated in a single institution. Clinical and pathological characteristics (tab. I) were used to subdivide all cases into two groups, i.e. (A) - 109 cases with low, and (B) - 106 cases with intermediate or high risk of local and/or distant failure (risk factors: poorly differentiated, positive estrogen/progesterone, HER-2 positive, extracapsular nodal involvement, too narrow surgical margins, regional nodal involvement). There were 162 cases with pT1 (75\%), and the remaining cases had $\mathrm{pT}$ 2. Similarly, a 3:1 ratio concerned the incidence of pN0 versus pN+. In the group A dominated pT1NO whereas pT2N+ in the group B. A higher rate (45\%) of pN+ was in group $B$, compared to a marginal rate (4\%) in group $A$.

\section{Methods}

\section{Treatment characteristics}

During CBS, all cases received intraoperative radiotherapy (IORT) performed using the INTRABEAM mobile device emitting low energy $\mathrm{X}$-rays (20-50 kV). Spherical applicators were tailored to the size of the postoperative tumor bed. The planned dose covered $0.5-1 \mathrm{~cm}$ of the tissue surrounding the postoperative margin, and usually a single dose of $5 \mathrm{~Gy}$

Table I. Clinical material characteristics

\begin{tabular}{|llll|}
\hline pT, N stage & $\begin{array}{l}\text { Group A } \\
(\mathbf{n}=109)\end{array}$ & $\begin{array}{l}\text { Group B } \\
(\mathbf{n}=106)\end{array}$ & $\begin{array}{l}\text { Overall } \\
(\mathbf{n}=215)\end{array}$ \\
\hline pT1 & $95-87 \%$ & $67-63 \%$ & $162-75 \%$ \\
\hline pT2 & $14-13 \%$ & $39-37 \%$ & $54-25 \%$ \\
\hline pN0 & $105-96 \%$ & $56-55 \%$ & $161-75 \%$ \\
\hline pN+ & $4-4 \%$ & $48-45 \%$ & $52-24 \%$ \\
\hline
\end{tabular}

was delivered. Since the RBE for X-rays of 20-50 kV is higher $(\sim 1.5-1.6)$ than that for high energy photons ( 1.0), an effective IORT single dose was 7.5 Gy. Postoperative EXRT used 50 Gy in 25 fractions. To estimate the izoeffective biological dose of these two different schedules, a Normalized Biological Effective Dose (NBED) was calculated using a Linear-Quadratic formula with $a / b$ value of $4.0 \mathrm{~Gy}$ :

$\mathrm{NBED}_{\text {TOTAL }}=\mathrm{RBE} \cdot \mathrm{NBED}_{\text {IORT }}+\mathrm{NBED}_{\text {EXT. }}$

$$
=14.4 \mathrm{izoGy}_{2.0}+50 \mathrm{izoGy}_{2.0}=64.4 \mathrm{izoGy}_{2.0}
$$

assuming that EXRT was delivered in the shortest possible time after completing the CBS.

\section{Adjuvant therapy}

Postoperative radiotherapy (EXRT) of $50 \mathrm{~Gy}$, given in $25 \mathrm{frac}-$ tions using 3D-IMRT as a sole adjuvant treatment was delivered to patients in group A. In group B, EXRT was combined with $\mathrm{CHT}$ as concurrent chemoradiation (with antracyclin, CTX and 5-Fu) in 62 cases (58\%), and in the remaining 42\%, EXRT was delayed after completing the $\mathrm{CHT}$. Therefore, the time interval (TI) between CBS (IORT) and adjuvant therapy widely differed between group $A$ and $B$. In group $A$, the TI was in the range of $10-45$ days, whereas in $74 \%$ of the $B$ cases, TI was delayed over 90 days, and in $40 \%$ of cases it was even longer than 120 days.

Hormonotherapy (tamoxifen) was additionally administered to $75 \%$ of patients and continued up to 5 years after completing combined therapy.

\section{End-points}

A ten-year follow-up was the only end-point to estimate the incidence of local recurrence free (LRFS) and distant metastases-free (DMFS) survival. The relationships between the accumulated incidence of $L$ R and DM during the follow-up and the sequence of adjuvant treatment, including the TI between CBS (IORT) and the EXRT, were counted using the Spearman correlation, the multivariate Cox' regression analysis and the $\mathrm{t}$-Student test modified by Yates. An estimate of $\mathrm{p}<0.05$ was accepted as a level of significance.

\section{Results}

The actuarial 10-year local tumor control (LTC) was $94.4 \%$ (97.1\% in gr. A and $91.6 \%$ in gr. B), and disease-free survival (DFS) of $84.8 \%$ (93.5\% in gr. A and $74.2 \%$ in gr. B).

\section{Incidence and kinetics of local and distant failures}

There were 12 local recurrences (LR - 5.6\%) and 21 distant metastases (DM - 9.7\%) during the 10-year follow-up. Although overall incidence of the LR or DM was not high, the LR rate in group $B$ doubled when compared with group $A$; the DM incidence in group $B$ was 4-times higher than in group $A$. Accumulation of the LR and the DM rates during the 10-year follow-up did not regularly and gradually increase, what is unusual attribute of these events. On the contrary, two-phase 
Table II. Number, overall and accumulated rates of Local recurrences and distant metastases during three time intervals within 10-year follow-up

\begin{tabular}{|c|c|c|c|c|}
\hline \multirow[t]{2}{*}{ Factors } & \multicolumn{3}{|c|}{ Follow-up in years } & \multirow{2}{*}{$\begin{array}{c}\text { Significance } \\
\text { (p) }\end{array}$} \\
\hline & $1-5$ & $6-7$ & $8-10$ & \\
\hline \multicolumn{5}{|l|}{ local recurrence } \\
\hline number (No.) & 8 & 1 & 3 & $<0.005$ \\
\hline overall rate & $3.7 \%$ & $0.5 \%$ & $1.4 \%$ & - \\
\hline accumulated rate & $67 \%$ & $75 \%$ & $100 \%$ & - \\
\hline \multicolumn{5}{|l|}{ distant metastases } \\
\hline number (no.) & 9 & 2 & 10 & $<0.0001$ \\
\hline overall rate & $4.1 \%$ & $0.9 \%$ & $4.7 \%$ & \\
\hline accumulated rate & $43 \%$ & $52 \%$ & $100 \%$ & \\
\hline
\end{tabular}

accumulation curves representing both types of failure were noted. The first phase concerns the 5-year follow-up, during which the accumulated rate of the LR gradually increased to $67 \%$, and the DM to $43 \%$ (tab. II).

During the next two years ( $6^{\text {th }}$ and $\left.7^{\text {th }}\right)$, the LR and DM rates slowed down, showing more or less a "plateau effect", and during the $8^{\text {th }}$ to $10^{\text {th }}$ year the accumulated rate of the LR steeply increased by $25 \%$, and the DM by $48 \%$. It seems that such an irregular accumulation rate of both failures might be explained by the biological and prognostic inhomogeneity of the two groups of patients with low vs. intermediate or high risk, and by the different aggressiveness of combined treatment modalities, lower in group A, and higher in group B.

\section{The impact of the time interval (TI) between CBS (IORT) and adjuvant therapy on treatment outcomes}

In 82 cases (75\%) in group A, the TI ranged from 10 to 45 days, and in the remaining 27 cases (25\%) the postoperative EXRT was delayed to over 60 days (for unknown reasons). On the contrary, in 28 cases (26\%) in group B, the TI was shorter than 60 days (all received concurrent chemoradiation). Therefore, the $\mathrm{Tl}$ of 60 days was arbitrarily chosen as the "TI cut-off limit". Table III shows that the duration of the TI had significant $(p<0.005)$ impact on the incidence of the LR, which in group $A$ and $B$ did not occur if the TI was shorter than 40 days (fig.1). However, the kinetics of the DM differed. For the TI up to 60 days it was low (1.2\%) in group A, but in group B it was much higher, up to $14 \%$. When the TI gradually extended above 80 days, the incidence of the LR and the DM significantly $(p<0.001)$ increased, being much higher in group B than A.

Extension of the $\mathrm{Tl}$ in group $\mathrm{B}$ depended on whether EXRT was delivered concurrently with $\mathrm{CHT}$ or was delayed after completing $\mathrm{CHT}$. The retrospective character of the analysis does not explain the reason for the two different ways of treatment decision. The delayed EXRT after completing CHT resulted in a 5 -fold higher incidence of the LR $(p<0.005)$ and more than doubled incidence of the DM ( $p<0.001)$. Figure 1 suggests that postoperative adjuvant therapy should begin as soon as possible but not later than 40-50 days after completing CBS $(\mathrm{IORT})$. Furthermore, for patients in group B (intermediate or high risk) optimally effective is concurrent CHT-EXRT, which lower the risk of both the LR and the DM (tab. IV).

Table III. Incidence of local recurrences (LR) and distant metastases (d. meta) in the group A and B depending on time interval (TI) between CBS (IORT) and adjuvant EXRT. For TI 60 days was accepted as border time

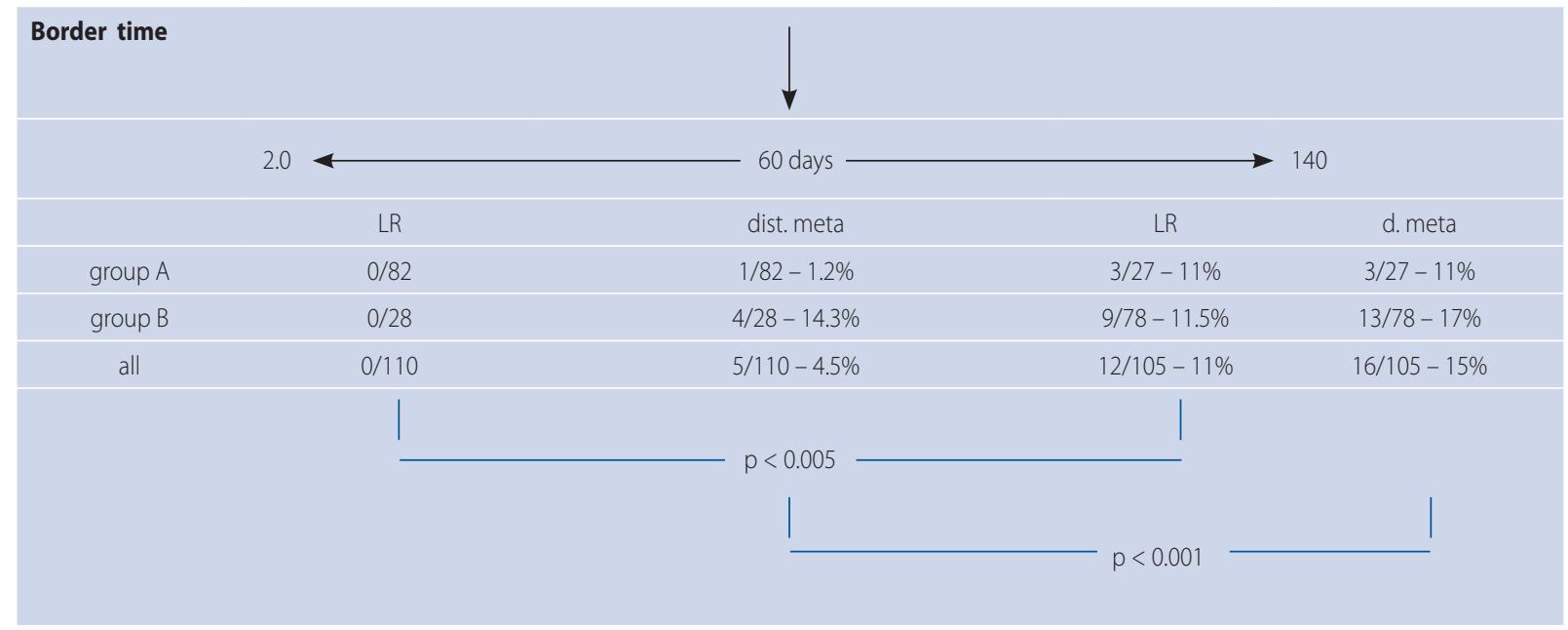



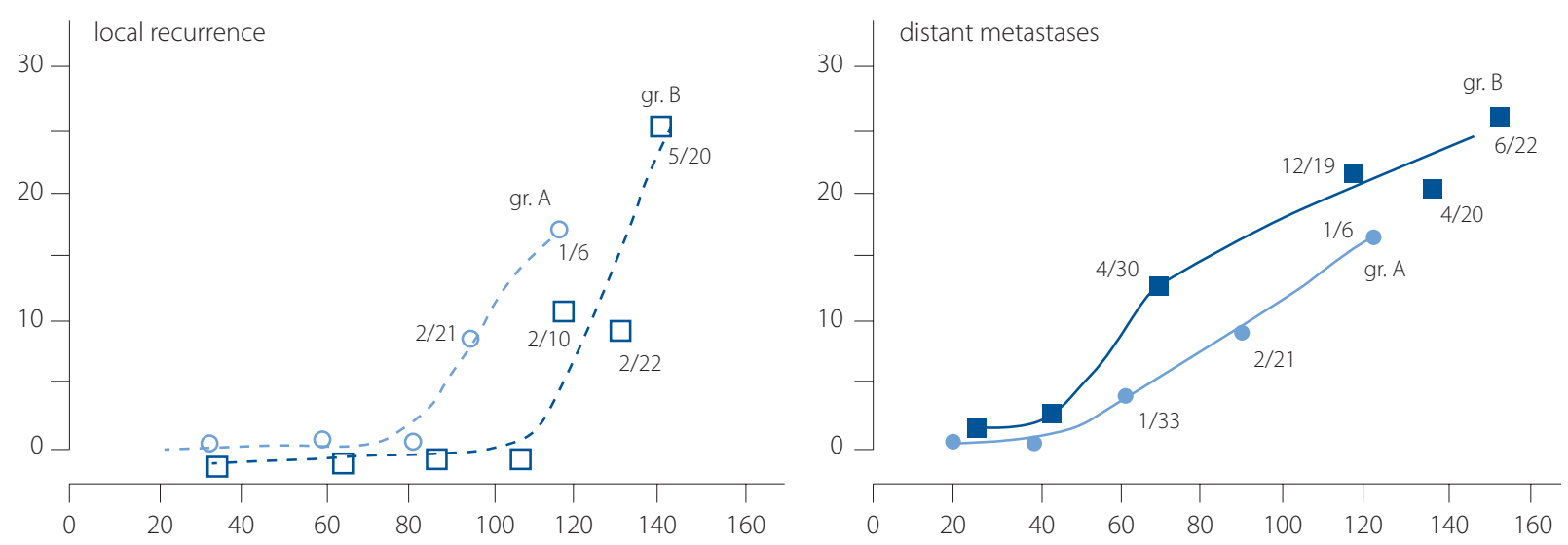

time interval between CBS (IORT) and EXERT in days

Figure 1. Incidence of local recurrence (LR) and distant metastases (DM) in the group A and B depending on time interval (TI) between CBS (IORT) and EXRT

Table IV. 10-year failure rates depending on the sequence of postoperative combined therapy in the group $B$

\begin{tabular}{lcc|} 
Sequence of postoperative $\begin{array}{c}\text { Sailures during } \\
\text { therapy }\end{array}$ & LR & dist. mear follow-up \\
\cline { 2 - 3 } & $2 / 62-3 \%$ & $6 / 62-10 \%$ \\
\hline concurrent cht-exrt & $7 / 44-16 \%$ & $12 / 4-27 \%$ \\
\hline cht followed by exrt & $<0.005$ & $<0.001$ \\
\hline significance $(p)$ & &
\end{tabular}

Multivariate analysis (tab. V) shows that $\mathrm{pN}$ is the strongest risk factor of the DM together with the number of involved regional nodes. If their number increases, i.e. from 1 to 5 positive nodes, the DM-HR (hazard ratio) becomes about 2.5-times higher (3.65/1.36 - see the bottom of tab. V). The highest LR risk factor strongly correlated with the EXRT delayed after completing CHT (HR = 14.28). If the TI between CBS (IORT) was 80 days (20 days longer than the TI cut-off limit of 60 days), HR increases to $1.02^{20}=1.485$, which means an increase of the LR and the DM by about 48\%, compared with that related to the TI of 60 days or less. On the contrary, concurrent chemoradiation resulted in significant $(p<0.001)$ decrease of the LR risk by about $94 \%$ and the DM risk by $66 \%$. The OTT of the EXRT was more or less the same (about 35 days) in all cases, therefore its prognostic power can be neglected, but the time interval between CBS (IORT) and the start of the postop. EXRT seems to be the major determinant of the LR and DM risk, mainly in group $B$, if the EXRT was considerably delayed after completing the $\mathrm{CHT}$.

\section{Discussion}

Since Veronesi [8] and Bartelink [9, 10] convincingly documented that early advance breast cancer patients need adjuvant radiotherapy after CBS, it was recognized that EXRT alone is not effective enough (7-15\% LR), especially for intermediate or high risk patients (11-15\%). It became obvious that conventional adjuvant EXRT needs a boost dose. Intraoperative radiotherapy using a single dose became an interesting solution. Although the use of IORT and adjuvant EXRT in the early

Table V. Results of multivariate Cox'regression analysis of the LR and DM risk factors

\begin{tabular}{|c|c|c|c|c|}
\hline \multirow[t]{2}{*}{ Factors } & \multicolumn{2}{|c|}{ LR } & \multicolumn{2}{|c|}{ DM } \\
\hline & HR & (p) & HR & (p) \\
\hline pT & 1.95 & 0.021 & 2.28 & 0.014 \\
\hline $\mathrm{pN}$ & 2.6 & 0.001 & 4.75 & 0.0004 \\
\hline number of involved regional nodes (x) & $1.18^{x}$ & 0.32 & $1.36^{x}$ & 0.0001 \\
\hline time interval (TI) between IORT and EXRT & 4.83 & 0.013 & 1.29 & 0.62 \\
\hline delayed time of EXRT after IORT above 60 days ( $n$ - days $>60$ ) & $1.02^{n}$ & 0.0008 & $1.02^{n}$ & 0.0007 \\
\hline concurrent CHT-EXRT & $0.07^{\bullet}$ & 0.0014 & $0.34^{\bullet}$ & 0.035 \\
\hline EXRT delayed after completing CHT & 14.28 & 0.001 & 2.94 & 0.035 \\
\hline
\end{tabular}

$\left[{ }^{x}\right.$ - if $x=1$ then $H R=1.18$, but for $x=5, H_{L R}=(1.18)^{5}-1=1.29$ and $H R_{D M}=(1.36)^{5}=3.65$

${ }^{n}$ - if TI increases by 20 days above $60 \mathrm{~d}$ limit then $H R=(1.02)^{20}=1.485$ means an increase of $L R / D M$ by $48.5 \%$ of that for $T \mid £ 60$ days

- - risk of $L R$ decreases by $93 \%(1-H R=1-0.07=0.93)$ and DM by $66 \%(1-H R=1-0.34=0.66)$ compared with those for sequential EXRT after CH] 
stages of breast cancer with no or low risk factors raised some doubts, Van Dongen et al. [16] and Clark et al. [17] strongly recommended IORT as a boost method preceding CBS. A recent comprehensive overview of the role of IORT in breast conserving therapy [18] provides the largest evidence of the high efficacy of the IORT with a single dose of 10-20 Gy, using electron beams. However, in the majority of the presented studies, the follow-up was shorter than 10 years (3-8 years). The IORT single dose of $14.4 \mathrm{izoGy}_{2.0}$ used in the present study is within the range of electron IORT doses of 10-20 Gy, due to the higher RBE of 20-50 kV X-rays, compared with that of electrons $(R B E=1.0)$. It is surprising that the recent ESTRO IORT TASK [18] aspires to recommend IORT but it raises some doubts and uncertainties. Local control and overall survival have been the only end-points and are related on average to only a 5-year actuarial follow-up, and the DM incidence has not been considered. The present study shows that beside the $L R$, the DM risk should not be ignored. Moreover, both failures accumulate nonlinearly during the follow-up (tab. II), and the 5-year follow-up is definitively too short, because about 30\% of the LR and $55 \%$ of the DM can easily be missed, since they may occur later mainly between the $7^{\text {th }}$ to $10^{\text {th }}$ year of the follow-up.

A recent ESTRO IORTTASK overview is focused on stage I-II breast cancer patients but no/low and intermediate/high risk patients are pulled together, whereas our study suggests that these two groups should be analyzed separately.

Bellon et al. [19] pointed out that sequence of $\mathrm{CHT}$ and EXRT for intermediate/high risk patients plays a very important role, but in the ESTROTASK overview, the time interval (TI) between IORT and adjuvant EXRT was not accounted, for similarly to Vaidya et al. $[20,21]$ in the TARGIT-A trial. On the contrary, multivariate analyses in the present study indicates the TI as the major determinant of the LR and DM risk. Considerable delay of the EXRT after completing postoperative $\mathrm{CHT}$ leads to the highest risk of the $L R(H R=14.28)$, even in the low risk group, where lengthening the TI over 60 days resulted in an increase of the LR from $0 \%$ to $11 \%$, and the DM from $1.2 \%$ to $11 \%$ (tab. III). A similar trend was also noted in group B. It may suggest that the TI lengthened over 60 days can completely ruin the efficacy of the IORT considered as a boost dose, and therefore, the necessity of its application might be questioned.

\section{Conclusions}

The present study suggests that the IORT as a part of conservative combined therapy for early stage breast cancer patients can be an effective boost, but only when the time interval (TI) between the IORT and EXRT is as short as possible. For intermediate/high risk patients, concurrent chemoradiation is highly advantageous to the CHT followed by the EXRT because this sequence lengthens the TI between the IORT and EXRT, and therefore it wastes therapeutic efficacy of the IORT as a boost dose. Finally, it seems that a 10-year follow-up should be considered as standard because in the shorter period (i.e. 5 years) about $40 \%$ of LR and even more DM can easily be missed.

Conflict of interest: none declared

\section{Bogusław Maciejewski}

Div. Research Programmes

M. Skłodowska-Curie National Research Institute of Oncology

Gliwice Branch

ul. Wybrzeże Armii Krajowej 15

44-102 Gliwice, Poland

e-mail:boguslaw.maciejewski@io.gliwice.pl

Received: 8 Dec 2020

Accepted: 15 Dec 2020

\section{References}

1. Abe M, Takahashi M. Intraoperative Radiation Therapy. Procc IIIrd Int. Symp on Intraoperative Radiation Therapy. Philadelphia and Pergamon Press 1991.

2. Vaidya JS, Wenz F, Bulsara M, et al. TARGIT trialists' group. Risk-adapted targeted intraoperative radiotherapy versus whole-breast radiotherapy for breast cancer: 5-year results for local control and overall survival from the TARGIT-A randomised trial. Lancet. 2014; 383(9917): 603-613, doi: 10.1016/S0140-6736(13)61950-9, indexed in Pubmed: 24224997.

3. Veronesi $\mathrm{U}$, Orecchia $\mathrm{R}$, Maisonneuve $\mathrm{P}$, et al. Intraoperative radiotherapy versus external radiotherapy for early breast cancer (ELIOT): a randomised controlled equivalence trial. Lancet Oncol. 2013; 14(13): 1269-1277, doi: 10.1016/S1470-2045(13)70497-2, indexed in Pubmed: 24225155.

4. Reitsamer R, Sedlmayer F, Kopp M, et al. The Salzburg concept of intraoperative radiotherapy for breast cancer: results and considerations. Int J Cancer. 2006; 118(11): 2882-2887, doi: 10.1002/ijc.21727, indexed in Pubmed: 16381011.

5. Herskind C, Steil V, Kraus-Tiefenbacher U, et al. Radiobiological aspects of intraoperative radiotherapy (IORT) with isotropic low-energy $X$ rays for early-stage breast cancer. Radiat Res. 2005; 163(2): 208-215, doi: 10.1667/rr3292, indexed in Pubmed: 15658897.

6. Herskind C, Griebel J, Kraus-Tiefenbacher U, et al. Sphere of equivalence--a novel target volume concept for intraoperative radiotherapy using low-energy X rays. Int J Radiat Oncol Biol Phys. 2008; 72(5): 1575-1581, doi: 10.1016/j.jijrobp.2008.08.009, indexed in Pubmed: 19028280.

7. Herdskind C, Wenz F. Radiobiological aspects of intraoperative tumour-bed irradiation with low-energy X-rays (LEX-IORT). Trans Cancer Res. 2014; 3: 3-17.

8. Veronesi U, Luini A, Del Vecchio M, et al. Radiotherapy after breast-preserving surgery in women with localized cancer of the breast. N Engl J Med. 1993; 328(22): 1587-1591, doi: 10.1056/NEJM199306033282202, indexed in Pubmed: 8387637.

9. Bartelink $\mathrm{H}$, Horiot JC, Poortmans $\mathrm{P}$, et al. European Organization for Research and Treatment of Cancer Radiotherapy and Breast Cancer Groups. Recurrence rates after treatment of breast cancer with standard radiotherapy with or without additional radiation. $\mathrm{N}$ Engl J Med. 2001; 345(19): 1378-1387, doi: 10.1056/NEJMoa010874, indexed in Pubmed: 11794170.

10. Vrieling $C$, van Werkhoven $E$, Maingon $P$, et al. European Organisation for Research and Treatment of Cancer, Radiation Oncology and Breast Cancer Groups, EORTC Radiation Oncology and Breast Cancer Groups, European Organisation for Research and Treatment of Cancer Radiation Oncology and Breast Cancer Groups. Whole-breast irradiation with or without a boost for patients treated with breast-conserving surgery for early breast cancer: 20-year follow-up of a randomised phase 3 trial. Lancet Oncol. 2015; 16(1): 47-56, doi: 10.1016/S1470-2045(14)71156-8, indexed in Pubmed: 25500422.

11. Kraus-Tiefenbacher $\mathrm{U}$, Bauer L, Scheda A, et al. Intraoperative radiotherapy (IORT) is an option for patients with localized breast recurrences after previous external-beam radiotherapy. BMC Cancer. 2007; 7: 178, doi: 10.1186/1471-2407-7-178, indexed in Pubmed: 17854511.

12. Veronesi U, Cascinelli N, Mariani L, et al. Twenty-year follow-up of a randomized study comparing breast-conserving surgery with radical mastectomy for early breast cancer. N Engl J Med. 2002; 
347(16): 1227-1232, doi: 10.1056/NEJMoa020989, indexed in Pubmed: 12393819.

13. Bartelink $\mathrm{H}$, Horiot JC, Poortmans $\mathrm{PM}$, et al. Impact of a higher radiation dose on local control and survival in breast-conserving therapy of early breast cancer: 10-year results of the randomized boost versus no boost EORTC 22881-10882 trial. J Clin Oncol. 2007; 25(22): 3259-3265, doi: 10.1200/JCO.2007.11.4991, indexed in Pubmed: 17577015.

14. Holland R, Veling $S$, Mravunac $M$, et al. Histologic multifocality of tis, T1-2 breast carcinomas implications for clinical trials of breast-conserving surgery. Cancer. 1985; 56(5): 979-990, doi: 10.1002/1097-0142(19850901)56:5<979::aid-cncr2820560502>3.0.co;2-n.

15. Clarke M, Collins R, Darby S, et al. Early Breast Cancer Trialists' Collaborative Group (EBCTCG). Effects of radiotherapy and of differences in the extent of surgery for early breast cancer on local recurrence and 15-year survival: an overview of the randomised trials. Lancet. 2005; 366(9503): 2087-2106, doi: 10.1016/S0140-6736(05)67887-7, indexed in Pubmed: 16360786.

16. van Dongen JA, Voogd AC, Fentiman IS, et al. Long-term results of a randomized trial comparing breast-conserving therapy with mastectomy: European Organization for Research and Treatment of Cancer 10801 trial. J Natl Cancer Inst. 2000; 92(14): 1143-1150, doi: 10.1093/ jnci/92.14.1143, indexed in Pubmed: 10904087.
17. Clark RM, McCulloch PB, Levine MN, et al. Randomized clinical trial to assess the effectiveness of breast irradiation following lumpectomy and axillary dissection for node-negative breast cancer. J Natl Cancer Inst. 1992; 84(9): 683-689, doi: 10.1093/jnci/84.9.683, indexed in Pubmed: 1314910 .

18. Fastner G, Gaisberger C, Kaiser J, et al. ESTRO IORT Task Force/ACROP recommendations for intraoperative radiation therapy with electrons (IOERT) in breast cancer. Radiother Oncol. 2020; 149: 150-157, doi: 10.1016/j.radonc.2020.04.059, indexed in Pubmed: 32413529.

19. Bellon JR, Come SE, Gelman RS, et al. Sequencing of chemotherapy and radiation therapy in early-stage breast cancer: updated results of a prospective randomized trial. J Clin Oncol. 2005; 23(9): 1934-1940, doi: 10.1200/JCO.2005.04.032, indexed in Pubmed: 15774786.

20. Vaidya JS, Bulsara M, Baum M, et al. Long term survival and local control outcomes from single dose targeted intraoperative radiotherapy during lumpectomy (TARGIT-IORT) for early breast cancer: TARGIT-A randomised clinical trial. BMJ. 2020; 370: m2836, doi: 10.1136/bmj. m2836, indexed in Pubmed: 32816842.

21. Vaidya J, Bulsara M, Saunders C, et al. Effect of Delayed Targeted Intraoperative Radiotherapy vs Whole-Breast Radiotherapy on Local Recurrence and Survival. JAMA Oncol. 2020; 6(7): e200249, doi: 10.1001/ jamaoncol.2020.0249. 